\title{
Turtles of the genera Geoemyda and Pangshura (Testudines: Geoemydidae) lack differentiated sex chromosomes: the end of a 40-year error cascade for Pangshura
}

Sofia Mazzoleni ${ }^{1}$, Barbora Augstenová ${ }^{1}$, Lorenzo Clemente ${ }^{1}$, Markus Auer $^{2}$, Uwe Fritz ${ }^{2}$, Peter Praschag ${ }^{3}$, Tomáš Protiva ${ }^{4}$, Petr Velenský ${ }^{5}$, Lukáš Kratochvíl ${ }^{1}$, Michail Rovatsos ${ }^{\text {Corresp. } 1}$

1 Department of Ecology, Charles University, Prague, Czech Republic

2 Museum of Zoology, Senckenberg, Dresden, Germany

3 Turtle Island, Graz, Austria

4 landsnails.org, Prague, Czech Republic

5 Prague Zoological Garden, Prague, Czech Republic

Corresponding Author: Michail Rovatsos

Email address: michail.rovatsos@natur.cuni.cz

For a long time, turtles of the family Geoemydidae have been considered exceptional, because representatives of this family were thought to possess a wide variety of sex determination systems. In the present study, we cytogenetically studied Geoemyda spengleri and Geoemyda japonica and re-examined the putative presence of sex chromosomes in Pangshura smithii. Karyotypes were examined by assessing the occurrence of constitutive heterochromatin, by comparative genome hybridization and in situ hybridization with repetitive motifs, which are often accumulated on differentiated sex chromosomes in reptiles. We found similar karyotypes, distributions of constitutive heterochromatin and topology of tested repetitive motifs for all three species. We did not detect differentiated sex chromosomes in any of the species. For $P$. smithii, ZZ/ZW sex determination system, with differentiated sex chromosomes, was described more than 40 years ago, but this finding has never been re-examined and was cited in all reviews of sex determination in reptiles. Here we show that the identification of sex chromosomes in the original report was based on the erroneous pairing of chromosomes in the karyogram, causing over decades an error cascade regarding the inferences derived from the putative existence of female heterogamety in geoemydid turtles. 


\section{Turtles of the genera Geoemyda and Pangshura (Testudines:}

2 Geoemydidae) lack differentiated sex chromosomes: the end

3 of a 40-year error cascade for Pangshura

4

5 Sofia Mazzoleni ${ }^{1}$, Barbora Augstenová1, Lorenzo Clemente ${ }^{1}$, Markus Auer ${ }^{2}$, Uwe Fritz ${ }^{2}$, Peter

6 Praschag $^{3}$, Tomáš Protiva ${ }^{4}$, Petr Velenskýn ${ }^{5}$, Lukáš Kratochvíl ${ }^{1}$, Michail Rovatsos ${ }^{1}$

7

$8{ }^{1}$ Department of Ecology, Charles University, Prague, Czech Republic

9 2Museum of Zoology, Senckenberg Dresden, Germany

$10{ }^{3}$ Turtle Island, Graz, Austria

11 4landsnails.org, Prague, Czech Republic

12

13

14 Corresponding Author:

15

16

17

18

19

20

21

22

${ }^{5}$ Prague Zoological Garden, Prague, Czech Republic

Michail Rovatsos

Viničná 7, Prague, 12844, Czech Republic

Email address: michail.rovatsos@natur.cuni.cz

\section{Abstract}

For a long time, turtles of the family Geoemydidae have been considered exceptional, because representatives of this family were thought to possess a wide variety of sex determination systems. In the present study, we cytogenetically studied Geoemyda spengleri and Geoemyda japonica and re-examined the putative presence of sex chromosomes in Pangshura smithii. Karyotypes were examined by assessing the occurrence of constitutive heterochromatin, by comparative genome hybridization and in situ hybridization with repetitive motifs, which are often accumulated on differentiated sex chromosomes in reptiles. We found similar karyotypes, distributions of constitutive heterochromatin and topology of tested repetitive motifs for all three species. We did not detect differentiated sex chromosomes in any of the species. For P. smithii, ZZ/ZW sex determination system, with differentiated sex chromosomes, was described more than 40 years ago, but this finding has never been re-examined and was cited in all reviews of 
31 sex determination in reptiles. Here we show that the identification of sex chromosomes in the

32 original report was based on the erroneous pairing of chromosomes in the karyogram, causing 33 over decades an error cascade regarding the inferences derived from the putative existence of 34 female heterogamety in geoemydid turtles.

\section{Introduction}

37 Turtles exhibit different sex determination modes. Although it is still a matter of debate, the ancestral (Valenzuela \& Adams, 2011; Johnson Pokorná \& Kratochvíl, 2016) and most common sex determination mechanism in turtles is most likely environmental sex determination (ESD). Genotypic sex determination (GSD) evolved independently in five families (Chelidae, Emydidae, Geoemydidae, Kinosternidae, Trionychidae) (Valenzuela \& Adams, 2011; Badenhorst et al., 2013). Turtles of the family Geoemydidae (Old World pond turtles) are a fascinating model for the evolution of sex determination, because it has been reported that this large family with more than 70 species (Rhodin et al., 2017) includes lineages with ESD as well as GSD, with both male (XX/XY sex chromosomes) and female (ZZ/ZW) heterogamety (Valenzuela \& Adams, 2011).

ESD was reported for three geoemydid genera, namely Mauremys (including Chinemys) (Rhodin et al., 2017), Melanochelys and Rhinoclemmys, mainly based on skewed sex ratios of hatchlings incubated at different temperatures (Ewert et al., 2004). So far, cytogenetic examinations revealed XX/XY sex chromosomes only in the black marsh turtle Siebenrockiella crassicollis (Carr \& Bickham, 1986; Kawagoshi et al., 2012) and ZZ/ZW sex chromosomes only in the brown roofed turtle Pangshura smithii (Sharma et al., 1975). The XX/XY sex chromosomes of $S$. crassicollis are medium-sized, and have been assigned as the fourth pair of the karyogram. The sex chromosomes are heteromorphic and with gene content partially homologous to chicken (Gallus gallus) and Chinese softshell turtle (Pelodiscus sinensis) chromosome pair five (Kawagoshi et al., 2012). The Y chromosome is metacentric, and the $\mathrm{X}$ chromosome is submetacentric, with a prominent C-positive band, missing on the Y. Despite that the $\mathrm{X}$ and $\mathrm{Y}$ chromosomes differ in morphology and C-banding pattern, it seems that they share gene content extensively. Sex-specific regions were not detected after single-copy gene mapping (Kawagoshi et al., 2012). Therefore, we assume that these sex chromosomes are at an early stage of differentiation with a small sex-specific region. For P. smithii, ZZ/ZW sex chromosomes have 
62 been reported by Sharma et al. (1975) based on distinct chromosome morphology. For the 63 majority of species of the family Geoemydidae, the sex determination mode remains unstudied.

In the current investigation, we cytogenetically explored the brown roofed turtle $P$.

65

66

67

68

69

70

71

72

73

74

75

76

77

78

79

80

81

82

83

84

85

86

87

88

89

\section{Materials \& Methods}

\section{Samples and species verification}

smithii, the black-breasted leaf turtle Geoemyda spengleri and the Ryukyu black-breasted leaf turtle Geoemyda japonica (Rhodin et al., 2017). Geoemyda is especially interesting because it represents the sister taxon of Siebenrockiella, a genus with an evident male heterogametic sex chromosomes (Carr \& Bickham, 1986; Kawagoshi et al., 2012). In addition, the genus Geoemyda is phylogenetically nested in a major geoemydid clade containing also Pangshura (Spinks et al., 2004; Lourenço et al., 2013; Pereira et al., 2017), a genus with reported female heterogametic sex chromosomes (Sharma et al., 1975).

The pioneering studies by Nakamura $(1937 ; 1949)$ reported a chromosome number of $2 \mathrm{n}$ = 52 for G. spengleri, but karyotypes were not documented photographically. In addition, neither the sex of the examined turtles nor their geographical origin was reported. We assume that Nakamura $(1937 ; 1949)$ actually studied G. japonica, since the Japanese populations had the status of a subspecies of G. spengleri at that time (Yasukawa et al., 1992). Chaowen and coauthors (1998) studied later undoubtedly G. spengleri, using individuals originating from Hunan Province, China. Chaowen and co-authors (1998) applied classic cytogenetic methods and revealed also a karyotype of $2 n=52$ chromosomes. However, no other cytogenetic approach has been applied to Geoemyda yet. Besides the karyogram of $P$. smithii published by Sharma and coauthors (1975), no further cytogenetic studies exist for this species.

In the present study, we constructed karyograms for all three species and further explored their karyotypes by C-banding stain to reveal the distribution of constitutive heterochromatin. Furthermore, we examined the presence of differentiated sex chromosomes by comparative genome hybridization (CGH) and fluorescence in situ hybridization (FISH) with repetitive elements that often accumulate on sex chromosomes of reptiles, such as telomeric motifs, $(\text { GATA })_{8}$ microsatellite repeats and rDNA loci (Literman et al., 2014; Matsubara et al., 2016; Rovatsos et al., 2017a; Augstenová et al., 2018). 
92 Blood samples from four individuals of G. japonica, five individuals of G. spengleri and four 93 individuals of $P$. smithii (Table 1) were used for preparation of mitotic chromosome suspensions 94 and DNA isolation. All turtles are captive-bred or legally imported, and kept in Plzen Zoo 95 (Czech Republic), Prague Zoo (Czech Republic) or the Museum of Zoology, Senckenberg 96 Dresden (Germany).

97 Genomic DNA was extracted using a DNeasy Blood and Tissue Kit (Qiagen). We 98 amplified by PCR and sequenced the mitochondrial cytochrome $b$ gene (cyt $b$ ), in order to verify 99 the taxon and to provide a DNA-based identity of our cytogenetically examined material for 100 future comparison (for the same approach please see Koubová et al., 2014; Rovatsos et al., 101 2015a; Rovatsos et al., 2015b; Rovatsos et al., 2016a). Cyt $b$ was amplified by PCR using the 102 primers L14919 5'-AACCACGGTTGTTATTCAACT-3' and H16064 5'103 CTTTGGTTTACAAGAACAATGCTTTA-3' (Burbrink et al., 2000; de Queiroz et al., 2002). 104 The PCR reaction protocol consists of $20-80 \mathrm{ng}$ of DNA, $1 \mu \mathrm{l}$ of each primer ( $10 \mathrm{pmol} / \mu \mathrm{l}), 5 \mu \mathrm{l}$ 105 of 10× PCR buffer (Bioline), $2.5 \mu \mathrm{l}$ of $\mathrm{MgCl}_{2}$ (50 mM), $1 \mu \mathrm{l}$ of dNTPs (10 mM each), $0.5 \mu \mathrm{l}$ of 106 BioTaq DNA polymerase (5 $\mathrm{U} / \mu \mathrm{l}$, Bioline) and water up to final volume of $50 \mu \mathrm{l}$. The 107 amplification conditions were: $95{ }^{\circ} \mathrm{C}$ for $3 \mathrm{~min}$, followed by 35 cycles of $95{ }^{\circ} \mathrm{C}$ for $30 \mathrm{sec}, 50{ }^{\circ} \mathrm{C}$ 108 for $30 \mathrm{sec}$ and $72{ }^{\circ} \mathrm{C}$ for $1 \mathrm{~min}$, and a final step of $72{ }^{\circ} \mathrm{C}$ for $5 \mathrm{~min}$. The PCR products were 109 sequenced by Macrogen (Korea), and the obtained sequences deposited in GenBank. A BLAST 110 search (Altschul et al., 1990) was performed to compare our sequences with those previously 111 deposited in public databases.

\section{Chromosome preparation and staining}

114 Mitotic chromosome suspensions were prepared from all studied individuals using whole blood 115 cell cultures. For leukocyte cultivation, $100-300 \mu \mathrm{l}$ of blood samples were cultured at $30^{\circ} \mathrm{C}$ for a 116 week without $\mathrm{CO}_{2}$ supplementation in $5 \mathrm{ml}$ of DMEM medium (Gibco) enriched with $10 \%$ fetal 117 bovine serum (Gibco), $100 \mu \mathrm{g} / \mathrm{ml}$ lipopolysacharide (Sigma-Aldrich), $2 \mathrm{mM} \mathrm{L-glutamine}$ 118 (Sigma-Aldrich), 3\% phytohaemaglutinin M solution (Gibco), 100 units $/ \mathrm{ml}$ of penicillin and 100 $119 \mu \mathrm{g} / \mathrm{ml}$ of streptomycin (Gibco). Three hours before harvesting, $35 \mu \mathrm{l}$ of colchemid solution (10 $120 \mu \mathrm{g} / \mathrm{ml}$ stock solution, Roche) was added to the medium. Chromosome suspensions were obtained 121 according to the standard method, including an initial hypotonic treatment with $0.075 \mathrm{M} \mathrm{KCl}$ at 
$12237{ }^{\circ} \mathrm{C}$ for $30 \mathrm{~min}$ and four times fixation in 3:1 methanol/acetic acid solution. Chromosome 123 suspensions were stored in freezer for further use.

124 Chromosomal spreads were stained with Giemsa solution, and selected metaphases were 125 captured in a Provis AX70 (Olympus) fluorescence microscope, equipped with a DP30BW 126 digital camera (Olympus). Subsequently, karyograms were constructed using Ikaros karyotyping 127 software (Metasystems).

128 The distribution of constitutive heterochromatin was detected by C-banding (Sumner, 129 1972). The slides were aged at $55^{\circ} \mathrm{C}$ for $1 \mathrm{~h}$, then soaked successively in $0.2 \mathrm{~N} \mathrm{HCl}$ at room 130 temperature for $45 \mathrm{~min}$, in $5 \% \mathrm{Ba}(\mathrm{OH})_{2}$ solution at $45^{\circ} \mathrm{C}$ for $4-5 \mathrm{~min}$ and in $2 \mathrm{xSSC}$ for $1 \mathrm{~h}$ at 60 $131{ }^{\circ} \mathrm{C}$, with intermediate washes in distilled water, and finally stained with 4',6-diamidino-2phenylindole (DAPI) and mounted with antifade medium Vectashield (Vector Laboratories).

\section{Fluorescence in situ hybridization with probes for repetitive elements}

The probe to detect the topology of rDNA loci was prepared from a plasmid (pDm r.a51\#1) with an 11.5-kb insertion, encoding the $18 \mathrm{~S}$ and $28 \mathrm{~S}$ rRNA units of Drosophila melanogaster (Endow, 1982) and labeled with biotin-dUTP using a Nick Translation Kit (Abbott Laboratories).

The probe for telomeric motifs (TTAGGG) ${ }_{\mathrm{n}}$ was produced and labeled with biotin-dUTP in a single PCR reaction using the primers $(\text { TTAGGG })_{5}$ and $(\text { CCCTAA })_{5}$ without a DNA template (Ijdo et al., 1991). The probes for the detection of rDNA loci and telomeric motifs were ethanol-precipitated with sonicated salmon sperm DNA and subsequently resuspended in hybridization buffer (50\% formamide/2xSSC) (Rovatsos et al., 2015a; Rovatsos et al., 2017b).

The probe for the GATA microsatellite motif was synthesized by Macrogen (Korea) as $(\text { GATA })_{8}$ and labeled with biotin. Subsequently, $0.3 \mu \mathrm{l}$ of (GATA) $)_{8}$ biotin-labeled probe (100 $\mathrm{pmol} / \mathrm{ul}$ stock solution) was diluted in $10 \mu \mathrm{l}$ of hybridization buffer $(50 \%$ formamide, $20 \mathrm{xSSC}$, $10 \%$ sodium dodecyl sulphate, $10 \%$ dextran sulphate, $1 \mathrm{x}$ Denhard's buffer, $\mathrm{pH}=7$ ) per slide.

The preparation of chromosome spreads and probes, the hybridization conditions, the post-hybridization washes, the signal amplification and detection are explained in detail in (Rovatsos et al., 2015a). At least 20 metaphases per slide were captured to confirm the fluorescent signal. The pictures were collected in black and white and superimposed with colors. 
153

154 Comparative Genome Hybridization

155 To detect putative sex-specific chromosome regions, CGH was used according to our standard 156 protocol (Rovatsos et al., 2015a). In each species, equal amounts of male and female genomic 157 DNA (1 $\mu \mathrm{g}$ each) were labelled independently with biotin-dUTP and digoxigenin-dUTP, 158 respectively, using a Nick translation kit (Abbott Laboratories) and then mixed together. 159 Sonicated salmon sperm DNA was added and ethanol-precipitation was carried out overnight at $16020^{\circ} \mathrm{C}$. The labelled DNA was resuspended in hybridization buffer, denatured at $75^{\circ} \mathrm{C}$ for 10 min 161 and immediately chilled on ice for $10 \mathrm{~min}$ prior to hybridization. The slides with chromosomal 162 material were subsequently treated with RNase A and pepsin, fixed with 1\% formaldehyde, 163 dehydrated through an ethanol series, denatured in $70 \%$ formamide $/ 2 \mathrm{xSSC}$ at $75{ }^{\circ} \mathrm{C}$ for $3 \mathrm{~min}$, 164 dehydrated again and air-dried. Hybridization was performed at $37{ }^{\circ} \mathrm{C}$ for two or three days. 165 Post-hybridization washes were performed three times in $50 \%$ formamide $/ 2 \mathrm{xSSC}$ at $42{ }^{\circ} \mathrm{C}$ for 5 $166 \mathrm{~min}$ and twice in $2 \mathrm{xSSC}$ at room temperature for $5 \mathrm{~min}$. Afterwards, the slides were incubated in 167 $100 \mu \mathrm{l}$ of $4 \mathrm{xSSC} / 5 \%$ blocking reagent (Roche) at $37{ }^{\circ} \mathrm{C}$ for $30 \mathrm{~min}$ and then with $100 \mu \mathrm{l}$ of 4xSSC/5\% blocking reagent including avidin-FITC (Vector Laboratories) and anti-digoxigenin rhodamine (Roche) at $37{ }^{\circ} \mathrm{C}$ for $30 \mathrm{~min}$. The slides were washed in $4 \mathrm{xSSC} / 0.05 \%$ Tween 20 , dehydrated, air dried, stained with DAPI, and mounted with Vectashield (Vector Laboratories).

\section{Results}

\section{Species verification}

The mitochondrial cyt $b$ gene was successfully amplified by PCR and sequenced in all three examined species. A BLAST search (Altschul et al., 1990) of the obtained sequences verified the expected taxonomic identity of the turtles examined here as G. japonica, G. spengleri and $P$. smithii. The haplotypes are deposited in GenBank, under the accession numbers MK097237MK097240.

\section{Karyotype reconstruction and C-banding}

Both $G$. japonica and $G$. spengleri have a similar karyotype with $2 \mathrm{n}=52$ chromosomes composed by 12 pairs of macrochromosomes, gradually decreasing in size and 14 pairs of 
184 acrocentric (pairs 6, 7 and 11) (Figure 1). C-positive bands were identified in the centromeric 185 regions of almost all chromosomes. A prominent heterochromatic block has been detected in the 186 chromosome pair 12 in metaphases of both sexes in both species (Figure 1).

187 In addition, $P$. smithii has similar karyotype with $2 \mathrm{n}=52$, consisting of 12 pairs of bi188 armed macrochromosomes and 14 pairs of microchromosomes. C-positive heterochromatin is 189 detected in the centromeric regions of all chromosomes. An extensive accumulation of 190 constitutive heterochromatin is detected in pair 12 in both sexes. This chromosome pair seems to 191 be polymorphic in size in some individuals, but this polymorphism is not linked to sex. 192

Fluorescence in situ hybridization and comparative genome hybridization

194 The rDNA loci are located in the terminal position in a pair of microchromosomes in G. japonica 195 and $P$. smithii, and near the centromere in a pair of microchromosomes in G. spengleri (Figure 196 197 198 2). The rDNA loci seem to be in all three species linked to the chromosome pair 12, which has the prominent $\mathrm{C}$-positive heterochromatic block.

199

The telomeric repeats $(\mathrm{TTAGGG})_{\mathrm{n}}$ showed the expected terminal chromosome topology in all studied individuals (Figure 2). The (GATA) $)_{8}$ microsatellite motif had a widespread 200 distribution in several pairs of microchromosomes and in the centromeric region of a pair of acrocentric macrochromosomes in both species of the genus Geoemyda, but without any sex202 specific signal. A weak signal of the (GATA) 8 microsatellite motif was detected in the telomeric 203 regions of several chromosomes in $P$. smithii, but without any sex-specific accumulation. Comparative genome hybridization did not reveal any sex-specific differences in any of the three 205 species (Figure 2).

206

\section{Discussion}

208 Our results confirmed that all three studied species have similar karyotypes with $2 \mathrm{n}=52$ 209 chromosomes, which agrees with former studies (Nakamura 1949; Sharma et al., 1975; 210 Killebrew 1977; Yasukawa et al., 1992; Chaowen et al., 1998). Geoemyda is phylogenetically 211 close to two lineages (Pangshura, Siebenrockiella) (Spinks et al., 2004; Lourenço et al., 2013; 212 Pereira et al., 2017) for which differentiated sex chromosomes have been reported (Carr \& 213 Bickham, 1986; Sharma et al., 1975; Kawagoshi et al., 2012). In several non-avian reptiles, 214 differentiated sex chromosomes are often highly conserved across the phylogenetic spectrum, 
215 e.g. in trionychid turtles (Rovatsos et al., 2017b), lacertids (Rovatsos et al., 2016b), iguanas 216 (Rovatsos et al., 2014) and caenophidian snakes (Rovatsos et al., 2015c). However, our 217 cytogenetic analysis using multiple approaches did not reveal any differentiated sex 218 chromosomes in G. spengleri and G. japonica. Thus, turtles of this genus have either GSD with 219 poorly differentiated sex chromosomes not detectable by our cytogenetic techniques, or ESD 220 where sex chromosomes are lacking (following the definition of ESD by Johnson Pokorná \& 221 Kratochvíl, 2016).

222 We did not detect sex chromosomes in $P$. smithii despite differentiated, highly 223 heteromorphic ZZ/ZW sex chromosomes had been shown by Sharma and co-authors (1975) in a 224 karyogram of this species using Giemsa-stained metaphase chromosomes. In this study, the Z 225 chromosome of $P$. smithii was identified as a small acrocentric, while the $\mathrm{W}$ as a medium-sized 226 metacentric chromosome. To explain the discrepancies between our results and those of Sharma 227 and co-authors (1975), we revisited their karyogram (Figure 3a) and we discovered several 228 potential errors in their assignment of chromosomes to homologue pairs that likely contributed to 229 the mischaracterization of $P$. smithii as possessing a ZZ/ZW system (Figure 3b). Namely, the 230 chromosome identified by Sharma et al. (1975) as the Z chromosome is a microchromosome, 231 and we conclude that it can be better reassigned as a homolog of one of the pairs 16 to 26. 232 Additionally, the metacentric chromosome identified by Sharma et al. (1975) as the W 233 chromosome could be reassigned as a homolog of pair 7, 8 or 9. After simple rearrangement of 234 the original karyogram, no obviously heteromorphic pair of chromosomes is detectable (Figure $2353 \mathrm{~b}$ ), consistent with our own karyotyping of new specimens (Figure 3c).

236 We found variability in size between the homologous chromosomes in the pair 12 in all 237 three examined species of turtles. This pair includes heterochromatic blocks co-localizing with 238 the accumulation of rDNA repeats (Figures 1,2). Heterochromatic blocks are often connected 239 with autosomal polymorphism due to rapid divergence of repeat numbers (Altmanová et al., 240 2016), and a polymorphism in chromosome morphology including rDNA genes was reported 241 also in ESD species of geoemydid turtles such as Rhinoclemmys pulcherrima (Carr \& Bickham 242 1986). The polymorphism of the chromosome pair 12 is not linked to sex in G. spengleri, G. 243 japonica or $P$. smithii, there is therefore no evidence that this pair correspond to sex 244 chromosomes. In any case, the chromosome pair 12 was not identified by Sharma et al. (1975) as 
245 sex chromosomes, although it might contribute to the incorrect pairing of chromosomes in their 246 karyotype (Figure 3).

247 According to our results, there is no evidence for female heterogamety with differentiated 248 sex chromosomes in geoemydid turtles of the genus Pangshura. Thus, among turtles, female 249 heterogamety is only known in softshell turtles (Trionychidae) (Badenhorst et al., 2013; 250 Rovatsos et al., 2017b). In the family Geoemydidae, the only reliable identification of sex 251 chromosomes refers to the XX/XY sex determination system of Siebenrockiella crassicollis 252 (Kawagoshi et al., 2012), while other studied species possess either ESD as most other lineages 253 of the family Geoemydidae with known sex determination (Figure 4) or, perhaps, GSD with 254 poorly differentiated and homomorphic sex chromosomes.

Unfortunately, the erroneous identification of putative sex chromosomes in P. smithii was 256 influential for scientific literature and impacted studies examining the cytogenetics of turtles 257 (Martinez et al., 2008; Kawagoshi et al., 2012) and comparative reconstructions as well as reviews of sex determination mechanisms, causing a 40-year error cascade regarding the inferred number of sex chromosome turnovers in amniotes and the evolution of sex determination and 260 genome organization (Modi \& Crews, 2005; Gamble 2010; Valenzuela \& Adams 2011; cascade caused by the putative sex chromosomes of $P$. smithii illustrates how little we still know about sex determination in reptiles and that even traditionally widely accepted reports of sex determining modes can benefit from re-examination with modern molecular cytogenetic methods and broader species sampling.

266

\section{Conclusions}

268 We found that G. spengleri, G. japonica and $P$. smithii share karyotypes with $2 \mathrm{n}=52$ 269 chromosomes and similar topology of constitutive heterochromatin and repetitive motifs. We did 270 not detect differentiated sex chromosomes in any of these species. It is particularly notable in $P$. 271 smithii, where ZZ/ZW sex determination system with differentiated sex chromosomes was 272 described more than 40 years ago. This information was repeated in subsequent reviews and 273 phylogenetic analyses on sex determination in amniotes and influenced their outcomes and 274 conclusions. We show that the identification of sex chromosomes in the original report was 275 based on the erroneous pairing of chromosomes in karyogram. We conclude that additional 
276 research is needed in order to clarify the true sex determination mode in the three studied species 277 of turtles, which might show either GSD with poorly differentiated sex chromosomes not 278 detectable by our cytogenetic techniques, or ESD as most other lineages of the family 279 Geoemydidae with known sex determination (Figure 4). The future directions of research should 280 include controlled incubation experiments of eggs to examine the influence of temperature in 281 hatchling sex ratios in G. spengleri, G. japonica and $P$. smithii, as well as cytogenetic 282 examination with molecular methods of additional geoemydid species, to better understand the 283 evolution of sex determination systems in this group.

284

\section{Acknowledgements}

286 We would like to express our gratitude to Petr Ráb for providing laboratory space and constant 287 support, to Nuria Viñuela Rodriguez and Jana Thomayerová for technical assistance. We thank 288 the staff of Prague Zoo and Zoo Plzeň for providing blood samples. We also thank Hidetoshi Ota 289 for providing Japanese literature and Christian Schmidt for translation to English.

290

291 References

292 Altmanová M, Rovatsos M, Kratochvíl L, Johnson Pokorná M. 2016. Minute Y 293 chromosomes and karyotype evolution in Madagascan iguanas (Squamata: Iguania: Opluridae). 294 Biol J Linnean Soc 118:618-633.

295 Altschul SF, Gish W, Miller W, Myers EW, Lipman DJ. 1990. Basic local alignment search 296 tool. J Mol Biol 215:403-410.

297 Augstenová B, Mazzoleni S, Kratochvíl L, Rovatsos M. 2018. Evolutionary dynamics of the 298 W chromosome in caenophidian snakes. Genes 9:5. http://dx.doi.org/10.3390/genes9010005

299 Badenhorst D, Stanyon R, Engstrom T, Valenzuela N. 2013. A ZZ/ZW microchromosome 300 system in the spiny softshell turtle, Apalone spinifera, reveals an intriguing sex chromosome 301 conservation in Trionychidae. Chromosome Res 21:137-147, https://doi.org/10.1007/s10577$302 \quad 013-9343-2$

303 Burbrink FT, Lawson R, Slowinski JB. 2000. Mitochondrial DNA phylogeography of the 304 polytypic North American rat snake (Elaphe obsoleta): a critique of the subspecies concept. 305 Evolution 54:2107-2118, https://doi.org/10.1554/0014-3820(2000)054[2107:mdpotp]2.0.co;2 
306 Carr JL, Bickham JW. 1986. Phylogenetic implications of karyotypic variation in the 307 Batagurinae (Testudines: Emydidae). Genetica 70:89-106.

308 Chaowen G, Ming W, Liuwang N. 1998. A cytogenetic study on three species of turtle. Acta 309 Hydrobiol Sin 1:002.

310 de Queiroz A, Lawson R, Lemos-Espinal JA. 2002. Phylogenetic relationships of North 311 American garter snakes (Thamnophis) based on four mitochondrial genes: How much DNA 312 sequence is enough? Mol Phylogenet Evol 22:315-329, https://doi.org/10.1006/mpev.2001.1074

313 Endow SA. 1982. Polytenization of the ribosomal genes on the $\mathrm{X}$ and $\mathrm{Y}$ chromosomes of 314 Drosophila melanogaster. Genetics 100:375-385.

315 Ewert MA, Etchberger CR, Nelson CE. 2004. Turtle sex-determining modes and TSD 316 patterns, and some TSD pattern correlates. In: Valenzuela N, Lance VA, eds. Temperature317 dependent sex determination in vertebrates. Washington D.C.: Smithsonian Institution Press, 21318 32, http://dx.doi.org/10.5479/si.9781944466213

319 Gamble T. 2010. A review of sex determining mechanisms in geckos (Gekkota: Squamata). Sex 320 Dev 4:88-103, https://doi.org/10.1159/000289578

321 Ijdo JW, Wells RA, Baldini A, Reeders ST. 1991. Improved telomere detection using a 322 telomere repeat probe (TTAGGG) generated by PCR. Nucleic Acids Res 19:4780, 323 https://doi.org/10.1093/nar/19.17.4780

324 Johnson Pokorná M, Kratochvíl L. 2016. What was the ancestral sex-determining mechanism 325 in amniote vertebrates? Biol Rev 91:1-12, doi:10.1111/brv.12156

326 Kawagoshi T, Nishida C, Matsuda Y. 2012. The origin and differentiation process of $X$ and $Y$ 327 chromosomes of the black marsh turtle (Siebenrockiella crassicollis, Geoemydidae, 328 Testudines). Chromosome Res 20:95-110, http://dx.doi.org/10.1007/s10577-011-9267-7

329 Killebrew F. 1977. Mitotic chromosomes of turtles. IV. The Emydidae. Tex J Sci 29:245-253.

330 Koubová M, Pokorná M.J, Rovatsos M, Farkačová K, Altmanová M, Kratochvíl L. 2014. 331 Sex determination in Madagascar geckos of the genus Paroedura (Squamata: Gekkonidae): are 332 differentiated sex chromosomes indeed so evolutionary stable? Chromosome Res 22:441-452, 333 https://doi.org/10.1007/s10577-014-9430-z

334 Literman R, Badenhorst D, Valenzuela N. 2014. qPCR-based molecular sexing by copy 335 number variation in rRNA genes and its utility for sex identification in soft-shell turtles. Methods 336 Ecol Evol 5:872-880, http://dx.doi.org/10.1111/2041-210X.12228 
337 Lourenço JM, Glémin S, Chiari Y, Galtier N. 2013. The determinants of the molecular 338 substitution process in turtles. J Evol Biol 26:38-50, http://dx.doi.org/10.1111/jeb.12031

339 Martinez PA, Ezaz T, Valenzuela N, Georges A, Graves JAM. 2008. An XX/XY 340 heteromorphic sex chromosome system in the Australian chelid turtle Emydura macquarii: a new 341 piece in the puzzle of sex chromosome evolution in turtles. Chromosome Res 16:815-825, 342 https://doi.org/10.1007/s10577-008-1228-4

343 Matsubara K, O’Meally D, Azad B, Georges A, Sarre SD, Graves JAM, Matsuda Y, Ezaz 344 T. 2016. Amplification of microsatellite repeat motifs is associated with the evolutionary 345 differentiation and heterochromatinization of sex chromosomes in Sauropsida. Chromosoma 346 125:111-123, https://doi.org/10.1007/s00412-015-0531-z

347 Modi WS, David C. 2005. Sex chromosomes and sex determination in reptiles. Curr Opin 348 Genet Dev 15:660-665.

349 Montiel EE, Badenhorst D, Tamplin J, Burke RL, Valenzuela N. 2017. Discovery of the 350 youngest sex chromosomes reveals first case of convergent co-option of ancestral autosomes in 351 turtles. Chromosoma 126:105-113, https://doi.org/10.1007/s00412-016-0576-7

352 Nakamura K. 1949. A study in some chelonians with notes on chromosomal formula in the 353 Chelonia. Chromosome 5:205-213.

354 Nakamura K. 1937. On the chromosomes of some chelonians (a preliminary note). Jap J Genet 355 13:240, https://doi.org/10.1266/jjg.13.240

356 Pereira AG, Sterli J, Moreira F.R, Schrago CG. 2017. Multilocus phylogeny and statistical 357 biogeography clarify the evolutionary history of major lineages of turtles. Mol Phylogenet Evol 358 113:59-66, http://dx.doi.org/10.1016/j.ympev.2017.05.008

359 Rovatsos M, Altmanová M, Johnson Pokorná M, Augstenová B, Kratochvíl L. 2017a. 360 Cytogenetics of the Javan file snake (Acrochordus javanicus) and the evolution of snake sex 361 chromosomes. J Zool Syst Evol Res 56:117-125, https://doi.org/10.1111/jzs.12180

362 Rovatsos M, Pokorná M, Altmanová M, Kratochvíl L. 2014. Cretaceous park of sex 363 determination: sex chromosomes are conserved across iguanas. Biol Lett 10:20131093, 364 http://dx.doi.org/10.1098/rsbl.2013.1093

365 Rovatsos M, Pokorná M.J, Altmanová M, Kratochvíl L. 2015a. Female heterogamety in 366 Madagascar chameleons (Squamata: Chamaeleonidae: Furcifer): differentiation of sex and neo367 sex chromosomes. Sci Rep 5:13196, https://doi.org/10.1038/srep13196 
368 Rovatsos M, Pokorná M.J, Altmanová M, Kratochvíl L. 2016a. Mixed-up sex chromosomes: 369 identification of sex chromosomes in the $\mathrm{X}_{1} \mathrm{X}_{1} \mathrm{X}_{2} \mathrm{X}_{2} / \mathrm{X}_{1} \mathrm{X}_{2} \mathrm{Y}$ system of the legless lizards of the 370 genus Lialis (Squamata: Gekkota: Pygopodidae). Cytogenet Genome Res 149:282-289, 371 https://doi.org/10.1159/000450734

372 Rovatsos M, Pokorná M.J, Kratochvíl L. 2015b. Differentiation of sex chromosomes and 373 karyotype characterisation in the dragonsnake Xenodermus javanicus (Squamata: 374 Xenodermatidae). Cytogenet Genome Res 147:48-54, https://doi.org/10.1159/000441646

375 Rovatsos M, Praschag P, Fritz U, Kratochvil L. 2017b. Stable Cretaceous sex chromosomes 376 enable molecular sexing in softshell turtles (Testudines: Trionychidae). Sci Rep 7:42150, 377 https://doi.org/10.1038/srep42150

378 Rovatsos M, Vukić J, Altmanová M, Johnson Pokorná M, Moravec J, Kratochvíl L. $2016 b$. 379 Conservation of sex chromosomes in lacertid lizards. Mol Ecol 23:3120-3126, 380 https://doi.org/10.1111/mec.13635

381 Rovatsos M, Vukić J, Lymberakis P, Kratochvíl L. 2015c. Evolutionary stability of sex 382 chromosomes in snakes. Proc R Soc B 282:20151992, https://doi.org/10.1098/rspb.2015.1992

383 Sharma GP, Kaur P, Nakhasi U. 1975. Female heterogamety in the Indian cryptodiran 384 chelonian, Kachuga smithi Gray. In: Tiwari KK, Srivistava CB, eds. Dr. B. S. Chuahah 385 Commemoration Volume. Orissa, India: Zoological Society of India, 359-368

386 Spinks PQ, Shaffer HB, Iverson JB, McCord WP. 2004. Phylogenetic hypotheses for the 387 turtle family Geoemydidae. Mol Phyl Evol 32:164-182, 388 https://doi.org/10.1016/j.ympev.2003.12.015

389 Sumner AT. 1972. A simple technique for demonstrating centromeric heterochromatin. Exp Cell 390 Res 75:304-306, https://doi.org/10.1016/0014-4827(72)90558-7

391 Rhodin AGJ, Iverson JB, Bour R, Fritz U, Georges A, Shaffer HB, van Dijk PP, TTWG392 Turtle Taxonomy Working Group. 2017. Turtles of the world: Annotated checklist and atlas 393 of taxonomy, synonymy, distribution and conservation status (8th edition). Chelon Res Monogr 394 7:1-202, doi:10.3854/crm.7.checklist.atlas.v8.2017

395 Valenzuela N, Adams DC. 2011. Chromosomes number and sex determination coevolve in 396 turtles. Evolution 65:1808-1813, doi:10.1111/j.1558-5646.2011.01258.x

397 Yasukawa Y, Ota H, Hikida H. 1992. Taxonomic re-evaluation of the two subspecies of 398 Geoemyda spengleri (Gmelin, 1792). Jpn J Herpetol 14:143-159. 


\section{Table $\mathbf{1}$ (on next page)}

Number of individuals per species and sex, analyzed in this study. 


\begin{tabular}{|c|c|c|}
\hline \multirow{2}{*}{ Species } & \multicolumn{2}{|c|}{ Sex } \\
\hline & $\hat{0}$ & q \\
\hline Geoemyda japonica & 2 & 2 \\
\hline Geoemyda spengleri & 3 & 2 \\
\hline Pangshura smithi & 2 & 2 \\
\hline
\end{tabular}

1 
Figure 1

Karyograms and C-banded metaphases of Geoemyda japonica (a-d), Geoemyda spengleri (e-h) and Pangshura smithii (i-l).

Please note that microchromosomes are paired according to size for illustration, which does not correspond to actual homology of chromosomes.
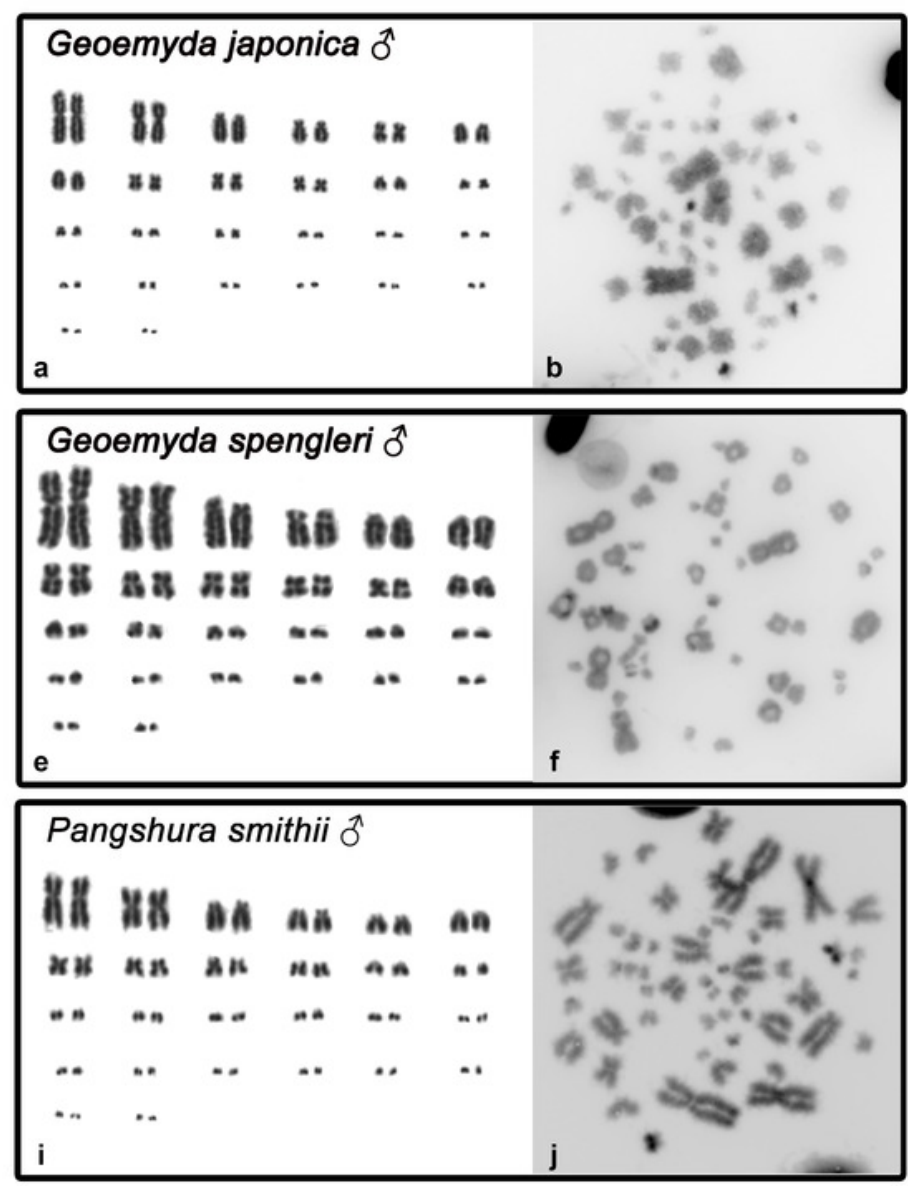
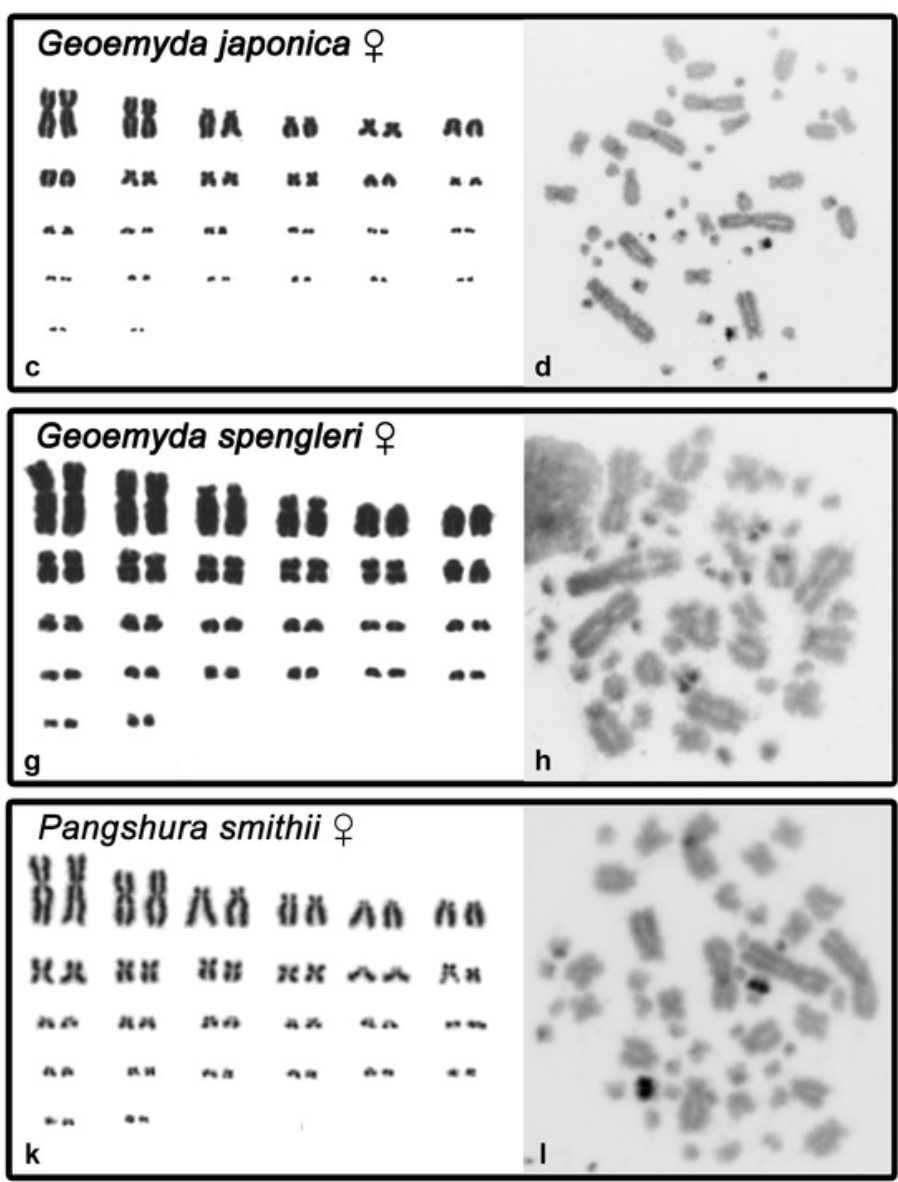


\section{Figure 2}

FISH with rDNA, (GATA) ${ }_{8}$ and telomeric probes in metaphases of Geoemyda japonica (ah), Geoemyda spengleri (i-p) and Pangshura smithii (q-x).

Chromosomes are stained blue with DAPI, and the signal of the probe is pseudocoloured in red. In CGH, the male genome is stained with FITC (green colour) and the female genome with rhodamine (red colour). Chromosomal regions with similar sequence content in both sexes are visualized in yellow. Arrows indicate the chromosome pair 12, with the prominent C-positive block. 


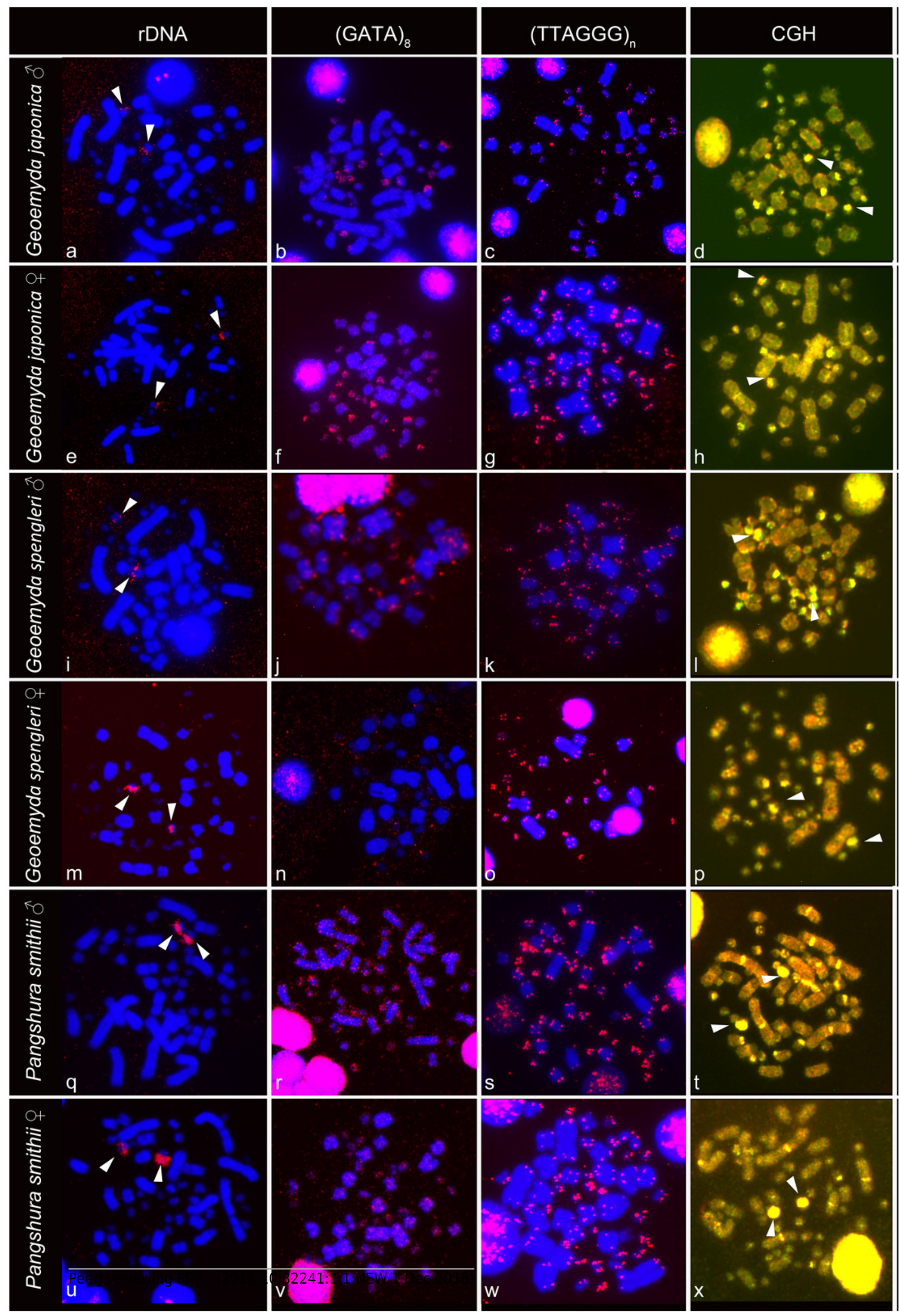




\section{Figure 3}

The original karyogram of Sharma and co-authors (1975) (a), their karyogram rearranged by us (b) and a new karyogram of a female individual from our studied material (c).

Note that the chromosomes misidentified as $Z$ and $W$ in the original study can be autosomal and easily assigned according to size and morphology into the pairs 16-26 and 7-9, respectively. Numbers in the re-arranged karyogram (b) refer to the original assignment of chromosome pairs by Sharma and co-authors (1975) . 

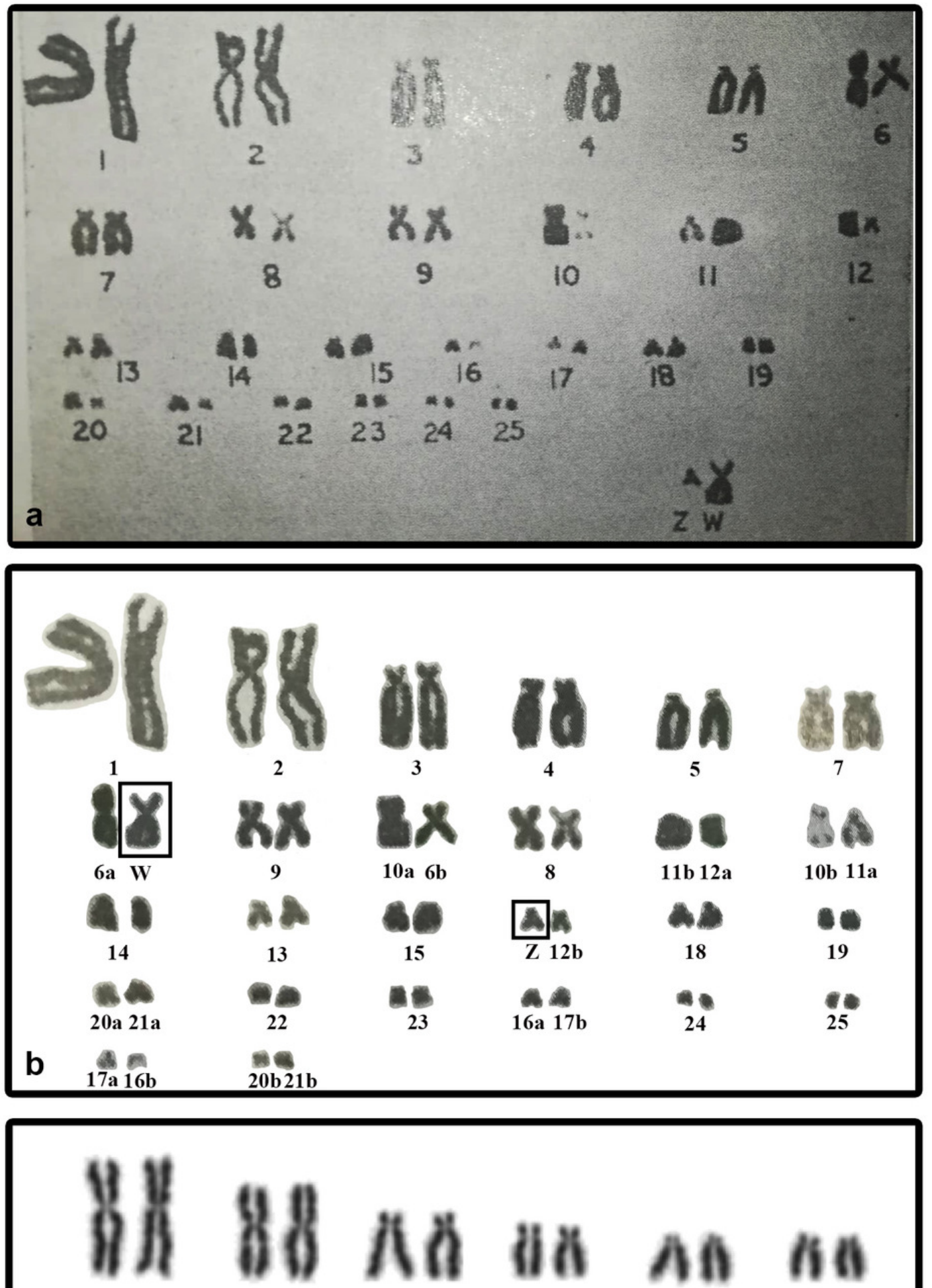

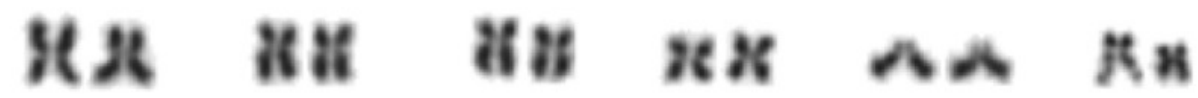

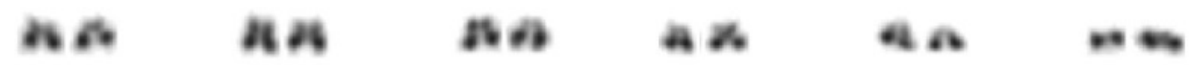

An wi na na




\section{Figure 4}

Phylogenetic reconstruction of the sex determination modes in turtles from the family Geoemydidae.

Phylogenetic relationships follow Spinks and co-authors (2004), Lourenço and co-authors (2013) and Pereira and co-authors (2017).

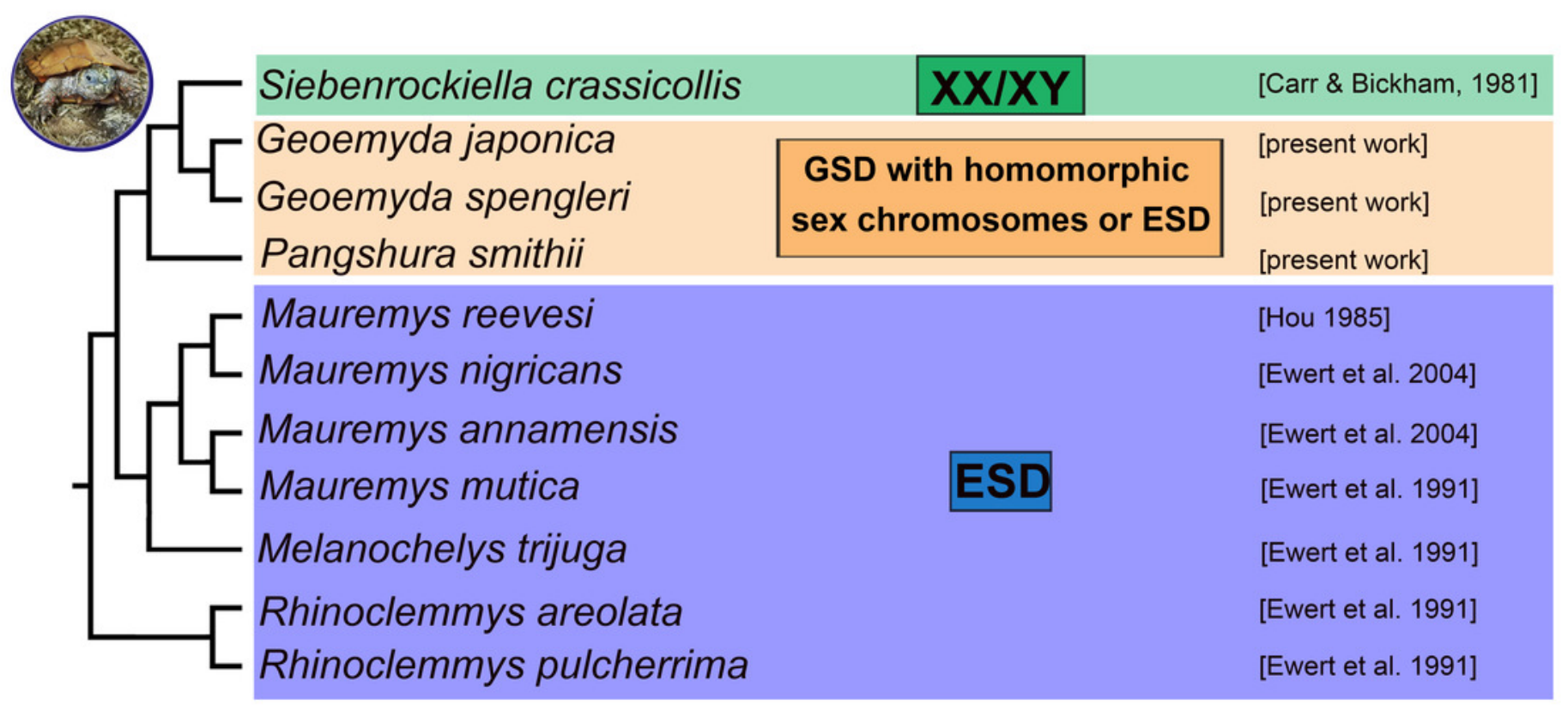

